\title{
Design of the ALPS II Optical System
}

\author{
M. T. Hartman, A. Lindner, R. C. G. Smith, A. D. Spector, and L.-W. Wei \\ Deutsches Elektronen Synchrotron (DESY), 22607 Hamburg, Germany
}

K. Karan, J. H. Põld, and B. Willke

Max-Planck-Institut fr Gravitationsphysik (Albert-Einstein-Institut) and Leibniz Universitt Hannover, 30167 Hannover, Germany

M. Diaz Ortiz, J. Gleason, A. Hallal, H. Hollis, T. Kozlowski, G. Messineo, G. Mueller, and D. B. Tanner

Department of Physics, University of Florida, 32611 Gainesville, Florida, USA

\author{
H. Grote and A. James \\ School of Physics and Astronomy, Cardiff University, CF24 3AA, Cardiff, United \\ Kingdom
}

October 1, 2020

\begin{abstract}
The Any Light Particle Search II (ALPS II) is an experiment currently being built at DESY in Hamburg, Germany, that will use a light-shining-through-a-wall (LSW) approach to search for axion-like particles. ALPS II represents a significant step forward for these types of experiments as it will use 24 superconducting dipole magnets, along with dual high-finesse, $122 \mathrm{~m}$ long optical cavities. This paper gives the first comprehensive recipe for the realization of the idea, proposed over 30 years ago, to use optical cavities before and after the wall to increase the power of the regenerated photon signal. This concept will allow the experiment to achieve a sensitivity to the coupling between axion-like particles and photons down to $g_{\alpha \gamma \gamma}=2 \times 10^{-11} \mathrm{GeV}^{-1}$ for masses below $0.1 \mathrm{meV}$, more than three orders of magnitude beyond the sensitivity of previous laboratory experiments. The layout and main components that define ALPS II are discussed along with plans for reaching design sensitivity. A set of top level requirements for the subsystems is also provided for the first time and includes the requirements on the coherence and spatial mode matching of the cavity eigenmodes. An accompanying paper (Hallal, et al [1]) offers a more in-depth description of the heterodyne detection scheme, the first of two independent detection systems that will be implemented in ALPS II.
\end{abstract}

*aaron.spector@desy.de 


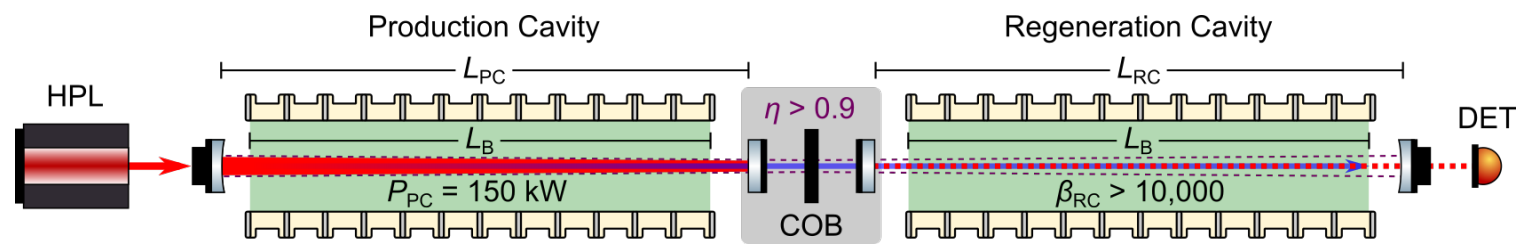

Figure 1: Experimental layout of ALPS II. Two $106 \mathrm{~m}$ long strings of twelve 5.3 T HERA dipoles are separated by the COB. The $\mathrm{COB}$ also houses the wall which blocks the PC transmitted light from reaching the RC. Each of the cavities are $122 \mathrm{~m}$ long. The power inside the $\mathrm{PC}$ should be at least $150 \mathrm{~kW}$, while the resonant enhancement of the $\mathrm{RC} \beta_{\mathrm{RC}}$, must be greater than 10,000 . The coupling efficiency between the $\mathrm{PC}$ and $\mathrm{RC}$, given by $\eta$, should be at least 0.9 .

\section{Introduction}

The Any Light Particle Search II (ALPS II) 2] will soon become the world's leading light-shiningthrough-a-wall (LSW) experiment. It will improve on the detection sensitivity to the coupling strength between photons and axion-like particles by three orders of magnitude compared to earlier experiments such as ALPS I [3] and OSQAR [4. Like all LSW experiments, ALPS II will directly probe the existence of pseudo-scalar fields whose coupling to electro-magnetic fields is described by the Lagrangian:

$$
\mathcal{L}_{a}=g_{a \gamma \gamma} \phi_{a} \vec{E} \cdot \vec{B}
$$

Here $g_{a \gamma \gamma}$ is the axion-photon coupling strength, $\phi_{a}$ is the axion field, the oscillating electric field is given by $\vec{E}$, and $\vec{B}$ represents the static magnetic field. This interaction is typically associated with the QCD or Peccei-Quinn axion, which was proposed to solve the strong CP problem [5, 6, 7]. In addition to the QCD axion, other 'axion-like' particles that can also be described by Equation 1 have recently taken centerstage. These particles, with potentially stronger interactions, offer possible explanations for a variety of astrophysical phenomena including the transparency of the universe to highly energetic photons [8] and anomalies in stellar cooling rates [9].

ALPS II will also be able to search for scalar fields whose coupling to electro-magnetic fields can be described by the Lagrangian:

$$
\mathcal{L}_{s}=g_{a \gamma \gamma} \phi_{s}\left(\vec{E}^{2}-\vec{B}^{2}\right)
$$

Experimentally, this only requires that the polarization of the E-field is orthogonal to the B-field. A signal running in both polarization modes with no observed polarization dependence on the production rate could be detected as well. This may indicate the existence of other types of Weakly Interacting Sub-eV Particles (WISPs) that are produced by kinetic mixing such as millicharged particles or hidden sector photons which do not require a static magnetic field to interact with photons [10]. In the following, we refer to particles whose interaction strength depends on $\vec{B}$ as axionlike particles, however the observation of any such particle would represent a profound discovery as it would be the first detection of an interaction beyond the standard model. If the new particle has a finite mass, depending on its type and interaction strength, it could also contribute to the total dark matter in the universe 11 .

It is worth pointing out that LSW experiments such as ALPS II make no assumptions regarding the natural prevalence of any of these particles, but merely probe the interactions themselves without the need for an external source. LSW experiments can therefore determine the photon-coupling strength independent of any astrophysical models, while solar searches and haloscopes, such as CAST [12, IAXO [13, ADMX [14, and MADMAX [15] not only depend on the coupling strength, but also rely on models of the axion-flux.

In contrast to these searches, LSW experiments take place entirely in the laboratory using a high-power laser (HPL) propagating through a magnetic field. This generates a beam of axion-like 
Table 1: Top level requirements of the ALPS II science run targeting a sensitivity of $g_{\alpha \gamma \gamma}=2.8 \times$ $10^{-11} \mathrm{GeV}^{-1}$ (adapted from 21]).

\begin{tabular}{|c|c|}
\hline & Requirement \\
\hline TLR1 & $150 \mathrm{~kW}$ power circulating in PC (fundamental mode, linearly polarized, $1064 \mathrm{~nm}$ ) \\
\hline TLR2 & $\begin{array}{l}\text { Parallel and perpendicular polarization adjustment possibility with respect to the mag } \\
\text { netic field }\end{array}$ \\
\hline TLR3 & Coupling between the axion mode and the RC fundamental mode: $\eta>90 \%$ (power ratio \\
\hline TLR4 & $\mathrm{RC}$ resonant enhancement $\beta_{\mathrm{RC}}>10000$ \\
\hline TLR5 & $\begin{array}{l}\text { Detector sensitive enough to confirm/exclude a reconverted photon rate of } 2.8 \times 10^{-5} / \\
\text { with a } 95 \% \text { confidence level within } 20 \text { days }\end{array}$ \\
\hline TLR6 & Magnetic field $\times$ length product of $560 \mathrm{~T} \cdot \mathrm{m}$ for PC and RC magnet string \\
\hline
\end{tabular}

particles that travel through a light-tight wall into a second magnetic field region where some of these axion-like particles convert back to photons [16.

ALPS II is based at DESY in Hamburg, Germany, taking advantage of the tunnels, magnets, and cryogenic infrastructure formerly used by the HERA accelerator. It will also use $122 \mathrm{~m}$ long optical cavities to resonantly enhance the electromagnetic field on both sides of the wall which increases the photon regeneration rate by twelve orders of magnitude over earlier LSW experiments [17, 18, 19, 20, The entire optical setup including these two cavities has to be tightly controlled to maintain and accurately calibrate the coupling of the generated axion field to the cavities.

This paper demonstrates the progress that has been made in the technical design for ALPS II and LSW experiments in general, as it includes the first detailed plan for maintaining both the coherence and transversal matching of the cavity eigenmodes. The following text will focus primarily on the core components that define optical system for the experiment and discuss how we plan to reach the targeted sensitivity, while also providing a set of top level requirements for the subsystems. A complementary paper [1], builds on the work presented here and describes the heterodyne detection scheme (HET) in more detail.

\section{$1.1 \quad$ ALPS II}

ALPS II will consist of two $122 \mathrm{~m}$ long, high-finesse optical cavities whose circulating fields will propagate through strings of 12 superconducting HERA dipole magnets [22, as shown in figure 1 . A current of $5.7 \mathrm{kA}$ will flow through these $8.8 \mathrm{~m}$ long dipoles and produce a magnetic field of $5.3 \mathrm{~T}$ giving a magnetic field times length of $B_{0} L=560 \mathrm{~T} \cdot \mathrm{m}$ on each side of the wall with free apertures between 46 and $51 \mathrm{~mm}$. Inside the production cavity $(\mathrm{PC})$ on the left side of the wall, photons will generate axion-like particles with an identical energy and spatial mode. These particles pass through the light-tight barrier on the central optical bench (COB) before they enter the regeneration cavity $(\mathrm{RC})$ where they convert back to photons. The regenerated photon rate,

$$
n_{\mathrm{reg}}=\frac{\eta}{16}\left(g_{a \gamma \gamma} F\left(q L_{\mathrm{M}}\right) B_{o} L\right) \frac{{ }^{4}}{P_{\mathrm{PC}}} \beta_{\mathrm{RC}},
$$

scales with $\left(g_{a \gamma \gamma} B_{0} L\right)^{4}$ and is proportional to the power $P_{\mathrm{PC}}$ inside the $\mathrm{PC}$ and the resonant enhancement $\beta_{\mathrm{RC}}$ of the RC [23]. The form factor can be approximated by the following equation with 
$L_{\mathrm{M}}$ representing the $106 \mathrm{~m}$ length of each magnet string.

$$
\begin{array}{r}
\left|F\left(q L_{\mathrm{M}}\right)\right| \approx\left|\frac{2}{q L_{\mathrm{M}}} \sin \left(\frac{q L_{\mathrm{M}}}{2}\right)\right| \\
\left(q=\frac{m_{a}^{2}}{2 \hbar \omega}\right)
\end{array}
$$

This is a typical phase matching condition which accounts for the possible mass $m_{a}$, of the relativisitic axion-like particles. For masses $m_{a}<0.1 \mathrm{meV}$, the form factor is essentially unity in ALPS II.

The coupling efficiency $\eta$ between the relativistic axion field and the eigenmode of the RC takes into account all transversal and spectral mismatches between the axion mode, which is identical to the PC eigenmode, and the eigenmode of the RC. Here $\eta$ is given in terms of axion to photon coupling and therefore the axion field to electromagnetic field coupling would be given by $\sqrt{\eta}$. It will be possible to verify $\sqrt{\eta}$ before and after measurement runs by opening a shutter in the light tight barrier and allowing the PC transmitted field to couple to RC.

Table 1 lists the top level requirements (TLR) of ALPS II. While the long magnet string (TLR 6) provides a sensitivity gain of $\sim 25$ when compared to ALPS I, TLR 1, requiring a PC internal power of $150 \mathrm{~kW}$, and TLR 4, requiring an RC resonant enhancement $\beta_{\mathrm{RC}}>10000$, together increase the sensitivity of the experiment by a factor of $\sim 40$, demonstrating the importance of the optical system to the experiment. Achieving both of these requirements depends on the coatings and surface roughnesses of the cavity mirrors as well as clipping losses in the magnet strings. This will be discussed further in section 2. It should be noted TLR 4 is not far from the limits of what is possible for mirrors of these dimensions with state of the art polishing techniques. TLR 3 refers to the coupling of the axion field to the RC and is discussed in section 3 . The current plan is to have an search based on the above listed parameters that could set an upper limit of $g_{\alpha \gamma \gamma}=2.8 \times 10^{-11} / \mathrm{GeV}$. This corresponds to a regenerated photon rate of $2.8 \times 10^{-5} / \mathrm{s}$ or roughly 2.4 photons per $24 \mathrm{~h}$ of valid data. This search will be followed by a scalar particle search at the same sensitivity by changing the polarization (TLR 2). We will then improve the sensitivity by increasing the PC circulating power, the RC resonant enhancement and the duty cycle to aim for $g_{\alpha \gamma \gamma}=2 \times 10^{-11} / \mathrm{GeV}$ or better [21] for pseudo-scalar and scalar particles.

\subsection{Detection Systems}

ALPS II will have the benefit of using two independent detection systems, each with very different systematic uncertainties, to measure the reconverted photons. This will help increase confidence in signals that are observed with the same strength in both detectors. The detectors themselves require different optical systems in order to be operated and cannot be used in parallel.

The first detection scheme to be implemented will be the HET, and its optical system is described in the accompanying paper in this journal 11. The HET utilizes an interference beatnote between a laser, referred to as the local oscillator (LO), and the regenerated photon field on a photodetector. Demodulating the electronic signal from this photodetector at the known difference frequency will create a signal proportional to the regenerated field strength that can be integrated over the measurement time. The regenerated photon signal will thus accumulate proportional to the measurement time $\tau$ while the laser shot noise will sum incoherently proportional to $\sqrt{\tau}$ [24].

A transition edge sensor (TES) will be used in the second detection system 25. The TES consists of an absorptive tungsten chip which is held at a temperature at the threshold of superconductivity. When a photon is incident on the chip it will be absorbed, leading to a slight increase in its temperature. This will suddenly raise the resistance of the chip causing a drop in the bias current that is flowing through it. This current drop can be measured with a superconducting quantum interference device. Therefore, the reconverted photons can be individually counted as these pulses occur, with an energy resolution of $\sim 5 \%$. 
Table 2: Parameters of the ALPS II cavities

\begin{tabular}{cc}
\hline \hline Parameter & Value \\
\hline Length & $122 \mathrm{~m}$ \\
Free spectral range & $1.2 \mathrm{MHz}$ \\
Half-width-half-maximum & $15 \pm 2.5 \mathrm{~Hz}$ \\
End mirror radius of curvature & $214 \pm 6 \mathrm{~m}$ \\
Waist radius $\left(1 / e^{2}\right)$ & $6.0 \mathrm{~mm}$ \\
End mirror beam radius $\left(1 / e^{2}\right)$ & $9.2 \mathrm{~mm}$. \\
Divergence half angle & $57 \mu \mathrm{rad}$ \\
RC resonant enhancement & $>10000$ \\
\hline
\end{tabular}

\section{ALPS II Cavities}

Both the PC and RC will be plano-concave cavities with $g=0.43$. The curved mirrors will be located at the end stations and the flat mirrors at the central station of the experiment as shown in Figure 2. The radius of curvature of the mirrors at the end stations were chosen such that the Rayleigh range of the cavity eigenmodes are equal to the length of the magnet strings. This geometry will help minimize aperture losses in the cavities while also avoiding higher order mode degeneracies that would occur if they were exactly half-confocal. The configuration also ensures that the eigenmodes of both cavities can have a high spatial overlap as the nominally identical Gaussian beam waists are located on the flat cavity mirrors on the COB. The distance between the flat cavity mirrors is $\Delta z \sim 835 \mathrm{~mm}$ and much smaller than the Rayleigh range $z_{R}$ of the modes. The resulting mismatch in power for $\Delta z \ll z_{R}$ is on the order of

$$
1-\eta_{\Delta z}=\left(\frac{\Delta z}{2 z_{R}}\right)^{2} \approx 10^{-5}
$$

which is negligible compared to other contributions to the total mismatch $\eta$.

The cavity eigenmodes will need to be centered within the beam tube of the magnet string to reduce clipping losses. The diameter of the beam tube is nominally $55 \mathrm{~mm}$, however since the magnets were originally used to steer protons around the arcs of the HERA accelerator, their central axis followed a curvature of $600 \mathrm{~m}$ and therefore required straightening. This process was very successful, and free apertures ranging from $46-51 \mathrm{~mm}$ were measured after being straightened. The magnets with the largest free apertures will be used near the end stations where the beam size and risk of clipping losses is the highest. The survey and magnet installation teams expect that they can place the magnets and the rest of the vacuum system housing the cavities to within $\pm 200 \mu \mathrm{m}$ and $\pm 1 \mu \mathrm{rad}$ of a line defining the theoretical optical axis of the experiment. The optics team then expects to be able to place the COB and cavity end mirror to within $\pm 1 \mathrm{~mm}$ and $\pm 8 \mu \mathrm{rad}$ of the resulting central line of the combined magnet string, sufficient to reduce clipping losses inside the two cavities to below $1 \mathrm{ppm}$.

\subsection{Regeneration cavity}

The resonant enhancement provided by the regeneration cavity,

$$
\beta_{\mathrm{RC}} \approx \frac{4 T_{\mathrm{out}}}{\left(T_{\mathrm{RC}_{1}}+T_{\mathrm{RC}_{2}}+\rho\right)^{2}},
$$

depends on the losses and transmissivities of each mirror. Here $T_{\text {out }}$ is the transmissivity of the mirror located nearest to the main regenerated photon detector; this will be $\mathrm{RC}_{2}$ for the $\mathrm{HET}\left(T_{\text {out }}=T_{\mathrm{RC}_{2}}\right)$ 

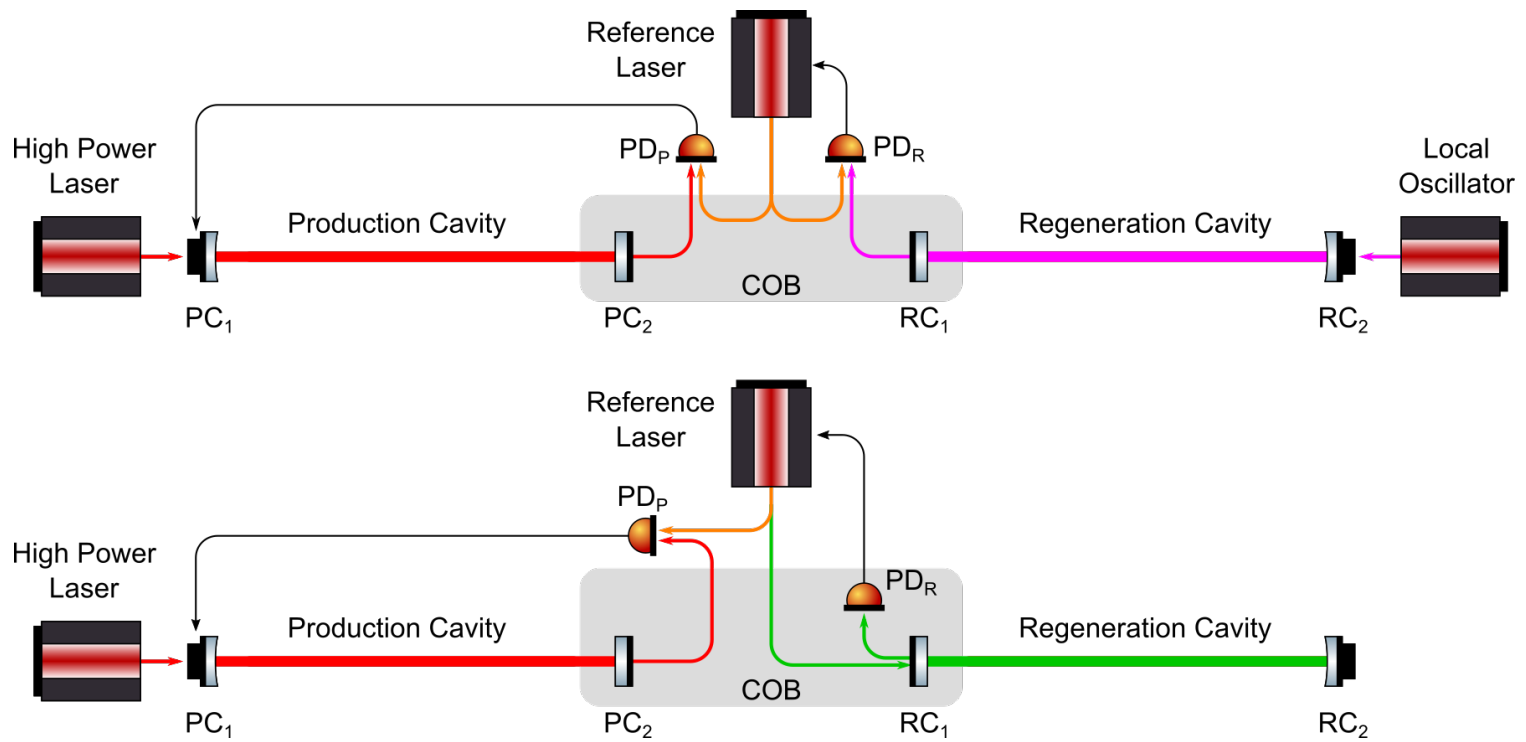

Figure 2: Layout of the cavities and control architecture for maintaining the phase lock of the PC in HET (top) and TES optical systems (bottom).

and is expected to be $\mathrm{RC}_{1}$ for the TES scheme $\left(T_{\text {out }}=T_{\mathrm{RC}_{1}}\right)$. Ideally, the maximum resonant enhancement occurs when the RC has minimal round-trip losses $\rho$, and the mirror transmissivities are as low as possible with the cavity still an impedance matched configuration where $2 T_{\text {out }}=$ $T_{\mathrm{RC}_{1}}+T_{\mathrm{RC}_{2}}+\rho$.

Losses in our cavities are expected to be dominated by the surface roughness of the mirrors and the associated scattering of light. We initially assumed that scatter losses can be kept below a few ppm per surface. Unfortunately, the fairly large beam sizes in relation to the size of the substrates together with the requirements on minimal wedge angles of the flat mirrors, proved to be a challenge for the polishing companies. After receiving the substrates, we estimated that scatter losses inside the cavity will likely be between 40 and $60 \mathrm{ppm}$ per round-trip. In addition to these losses, the HET also requires some transmission through $T_{\mathrm{RC}_{1}}$ to realize their sensing and control scheme as shown in Figure 2 [1]. To be conservative, we decided to use $100 \mathrm{ppm}$ as the design value for $T_{\mathrm{RC}_{2}}$ and $5 \mathrm{ppm}$ for $T_{\mathrm{RC}_{1}}$ for the HET. For the TES detection scheme we plan to flip these values such that $T_{\mathrm{RC}_{1}}$ will be $100 \mathrm{ppm}$ while $T_{\mathrm{RC}_{2}}$ will be $5 \mathrm{ppm}$; however, in order to optimize the sensitivity of the the experiment, the transmissivities of these mirrors for the TES system are subject to change based on what we learn from commissioning the HET optical system.

For the HET optical system the dielectric mirror coatings consist of alternating $\lambda / 4$ layers of silica and tantala to minimize the absorptive losses; note that the same coatings will also be used in the PC where laser beam absorption will lead to thermal distortions of the cavity eigenmode. These mirrors were received and measured to have transmissivities of $\sim 110 \mathrm{ppm}$ and $\sim 6.7 \mathrm{ppm}$ at normal incidence which results in an expected resonant enhancement of

$$
\beta \approx 16000 \pm 2000
$$

for losses between 20 and 30 ppm per mirror.

The design of coatings for the TES optical system are still being optimized, however preliminary designs make use of custom stacks that are capable of the reflecting light with at wavelengths of 1064 and $532 \mathrm{~nm}$, in addition to the $\lambda / 4$ stack which are optimized for $1064 \mathrm{~nm}$. This is critical to the TES related optical system as it will use green light to sense the length of the $\mathrm{RC}[2$. 


\subsection{Production cavity}

A consequence of the cavities natural amplification is that using the same mirrors the PC will give it a power build-up factor of $16000 \pm 2000$, the same as the resonant enhancement of the RC. The PC will be seeded with a linear polarized laser beam from a single frequency, low noise laser system operating at $1064 \mathrm{~nm}$. This system is based on a nonplanar-ring-oscillator (Coherent Mephisto 2000) amplified by a neoVAN 4S-HP amplifier (similar to [26]) giving a maximum output power of $70 \mathrm{~W}$. A frequency stabilization system with a control bandwidth of $\sim 300 \mathrm{kHz}$ will maintain the resonance of the laser with respect to the length of the PC using the standard Pound-Drever-Hall (PDH) technique [27, 28. The sensing scheme and the loop gain are expected to keep the laser frequency within a hundredth of the HWHM of the cavity resonance corresponding to a relative power noise inside the cavity to a RMS value of approximately $100 \mathrm{ppm}$.

The input optics between the HPL and the PC will also be equipped with an automatic alignment system based on a differential wavefront sensing (DWS) scheme. This system uses a pair of quadrant photodetectors (QPDs) which measure the lateral shift $\Delta x$ and angular offset $\Delta \theta$ between the laser mode and the cavity eigenmode [29]. We expect to reach sensitivities of:

$$
\Delta x_{\mathrm{RMS}}<0.01 \cdot w_{0} \quad \Delta \theta_{\mathrm{RMS}}<0.01 \cdot \theta_{\text {Div }}
$$

These signals are then fed back to a pair of actuators to maintain the alignment into the cavity. The goal is to also limit the RMS relative power noise inside the cavity due to alignment fluctuations to $100 \mathrm{ppm}$ for each degree of freedom. The entire system should guarantee that the total relative power noise stays below $0.1 \%$ RMS, which could be critical to reducing dynamic thermal effects from effecting the HET [1]. The input optics for the PC will also employ a half-waveplate before mirror $\mathrm{PC}_{1}$ to rotate the polarization of the circulating field with respect to the polarity of the magnet string. This will satisfy TLR2 and allow the experiment to search for both scalar or pseudo-scalar particles.

This combination of the HPL and cavity finesse may allow powers as high as $1 \mathrm{MW}$ inside the PC, however, the final power level will likely be limited by the absorption in the HR coating layers of the two cavity mirrors. There are a number of ways this absorbed light could lead to thermal effects that cause higher intracavity losses. For example, point absorbers heating up on the surface of the mirror could cause the formation of low spatial frequency features which, in turn, leads to an increase in the scattering losses [30, 31]. Absorption in the mirror coatings could also cause the size of the mode circulating in the PC to change and lead to additional clipping loses from the beam tube [32. The loss in sensitivity due to the mode mismatch between the cavity eigenmodes as the $\mathrm{PC}$ mirrors heat up is expected to be insignificant in comparison. In spite of these effects, we are confident that $150 \mathrm{~kW}$ is achievable.

\section{Maintaining the Axion Field Coupling to the RC}

The primary obstacles to optimizing the coupling of the axion field to the $\mathrm{RC}$ are related to maintaining the coherence and spatial mode matching between them. Both of these parameters will depend on the residual changes of the $\mathrm{PC}$ eigenmode with respect to the eigenmode of the $\mathrm{RC}$ and we allow each to contribute a $5 \%$ loss of the signal to meet the $90 \%$ coupling efficiency listed under TLR 3. Admittedly, this rather unsophisticated split is a reflection of our limited understanding of the expected mirror motion in the HERA tunnel once the optical tables, the vacuum system, the magnets and the clean rooms are all installed and operational.

\subsection{Coherence of the PC field with the RC}

Maintaining the coherence between the electromagnetic field regenerated from the axion field and the RC eigenmode is critical to ALPS II achieving its target sensitivity. Therefore, the regenerated 
field should experience no more than $5 \%$ average reduction from its optimal resonant enhancement over the duration of the measurement due to its frequency noise with respect to the length of the RC. This requirement is further divided into one on static frequency offset and one on the dynamic phase noise. As the regenerated field is a replica of the field circulating in the PC, the first challenge is to accurately tune the frequency of the $\mathrm{PC}$ transmitted field such that it is resonant with the RC. The second challenge is to precisely control the phase of the PC transmitted field around this nominal value.

\subsubsection{PC Tuning}

The signal loss in regenerated photons due to a small offset of $\Delta f$ in the frequency of the $\mathrm{PC}$ transmitted light relative to a resonance frequency of the RC is quadratic in the offset and can be approximated by the following expression.

$$
1-\eta_{\Delta f} \approx\left(\frac{\Delta f}{\mathrm{HWHM}}\right)^{2}
$$

Here HWHM is the half-width half-max linewidth of the RC. To limit the loss of regenerated photons to $1 \%$, we require that the detuning is less than $10 \%$ of the HWHM or less than $1.5 \mathrm{~Hz}$ during the science run.

As Figure 2 shows, in both detection systems the frequency of the PC transmitted field $\nu_{\mathrm{PC}}$ will be set via offset phase lock loops relative to a frequency of a reference field which itself is locked to a resonance frequency $\nu_{\mathrm{RC}}$ of the RC. The offset frequency, a multiple $N$ of the FSR of the $\mathrm{RC}$, is optimized by maximizing the transmission through the $\mathrm{RC}$ with the shutter open and then maintained during science runs when the shutter is closed [1]. This approach requires that the source for the offset frequency and the FSR of the RC are both stable.

The RF frequency will be derived from a clock that is synchronized to a $10 \mathrm{MHz}$ rubidium frequency standard with a yearly frequency drift on the order of $\mathrm{mHz}$, well below our requirement. However, macroscopic changes of the length of the RC will change the optimum offset frequency by:

$$
\Delta f_{\Delta \mathrm{FSR}}=N \cdot \Delta \mathrm{FSR}_{\mathrm{RC}}=N \cdot \mathrm{FSR}_{\mathrm{RC}} \frac{\Delta L_{\mathrm{RC}}}{L_{\mathrm{RC}}}
$$

The length changes of the $\mathrm{RC}$ then have to be

$$
\Delta L<\frac{\Delta f_{\Delta \mathrm{FSR}}}{\operatorname{FSR}_{\mathrm{RC}}} \frac{L}{N}=\frac{150 \mu \mathrm{m}}{N}
$$

between retuning measurements to ensure that $\Delta f<1.5 \mathrm{~Hz}$. We implement a FSR sensing system that uses a modified PDH sensing technique which uses phase modulated sidebands at some multiple $(\neq N)$ of the FSR [33. $\Delta L$ will be measured continuously during the science run and if it becomes larger than $\frac{150 \mu \mathrm{m}}{N}$ the run will be paused and the length of the RC will be adjusted back to its initial value before it is started again. We also are investigating options to actively control the length of the $\mathrm{RC}$ during a measurement run.

\subsubsection{Phase lock of the PC}

The feedback system which reduces the frequency or phase fluctuations $\phi(t)$ of the $\mathrm{PC}$ transmitted field relative to the offset frequency set by the phase lock loop has to provide the precision necessary to meet the requirements on the coherence. Phase noise in the PC transmitted field relative to the $\mathrm{RC}$ spreads the energy of the ideally monochromatic field over a finite frequency band and only the frequency components which are resonant in the $\mathrm{RC}$ will contribute to the signal. The energy in all frequency components outside the FWHM line-width of the RC will be attenuated. 
We require that the power integrated over all frequency components outside this bandwidth is less than $4 \%$ of the total power. This requirement roughly translates into an upper limit for the standard deviation (SD) of the phase noise evaluated over the storage time $\mathcal{T}$ of the cavity of [34:

$$
\Delta \phi_{\mathrm{SD}}(t) \approx \sqrt{\left\langle\delta \phi^{2}(t)\right\rangle_{\mathcal{T}}}<0.2 \mathrm{rad}
$$

which the phase lock loop between the PC transmitted field and the reference field has to achieve.

Due to the high gain and fast bandwidth of the control loop that stabilizes the HPL to the PC, the phase of the PC transmitted light is mostly determined by its length. The phase lock loop will have to act on the length of the $\mathrm{PC}$ such that it follows all length changes of the $\mathrm{RC}$ which are impressed on the phase of the reference laser as shown in Figure 2

We developed a piezo-electric actuated mirror mount for the 2" diameter $\mathrm{PC}_{1}$ mirror that supports a control bandwidth of $4 \mathrm{kHz}$. Based on seismic measurements in the HERA tunnel, this bandwidth paired with an aggressive gain function is expected to be sufficient to suppress the environmental noise 34 .

\subsection{Transversal matching}

Another effect which could lead to a loss in sensitivity is related to the alignment of the axion field into the RC. The spatial mode of the axion field entering the $\mathrm{RC}$ is an extension of the spatial mode inside the PC and the loss due to small alignment errors can be calculated from the following equation:

$$
1-\eta_{\mathrm{TM}} \approx\left(\frac{\delta x}{w_{0}}\right)^{2}+\left(\frac{\delta \theta}{\theta_{\text {Div }}}\right)^{2}
$$

where $\delta x$ is the transversal shift and $\delta \theta$ is the angular misalignment between the two modes measured at the waist of the RC. The power loss is quadratic in both terms and required to be less than $5 \%$ in total.

\subsubsection{Quantifying the transversal matching}

Like the tuning of PLL offset frequency, the alignment of the cavity eigenmodes will be quantified using the PC transmitted field when the shutter is open. This quantification has a systematic error due to the refraction in all optical components located between the cavity internal fields. The substrates of the cavity end mirrors have known wedge angles $\theta_{\mathrm{w}}$ between 3 and $4 \mu \mathrm{rad}$ which will refract the PC transmitted field but not the axion field. By clocking the two substrates correctly, the refraction angles will compensate each other such that the final angular refraction is:

$$
\delta \theta_{\text {refr }}=(n-1) \Delta \theta_{\mathrm{w}}<1 \mu \mathrm{rad}
$$

The refraction in $\mathrm{PC}_{2}$ will laterally shift the $\mathrm{PC}$ transmitted beam by $\delta x<4 \mu \mathrm{m}$.

Each of the detection systems uses additional beam splitters between the two cavities to direct the various laser beams required to operate the experiment 11. These beam splitters are also made from substrates with known wedge angles between 2 and 5 prad and will also be clocked to reduce the overall deflection to below 2 prad. The total deflection angle between the beams will therefore be below a 3 urad.

Each substrate will laterally shift the beam by:

$$
y=d \frac{\sin \left(\theta_{1}-\theta_{2}\right)}{\cos \theta_{2}} \quad \sin \theta_{1}=n \sin \theta_{2}
$$

where $d$ is the thickness of the substrate, $\theta_{1}$ the angle of incidence and $\theta_{2}$ the angle inside the material. In both designs, the number of substrates which shift the beam to the left has to equal 


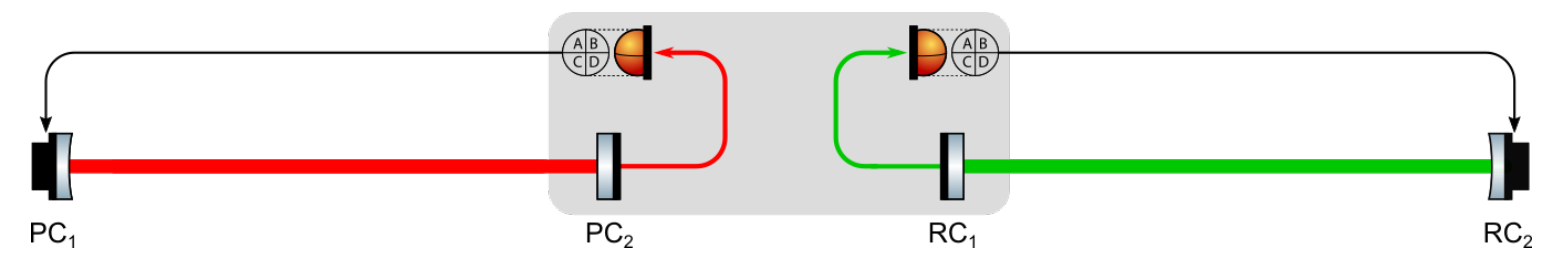

Figure 3: Control architecture for maintaining spatial overlap. The QPDs on the COB monitor the position of the cavity eigenmodes on the cavity mirrors $\mathrm{PC}_{2}$ and $\mathrm{RC}_{1}$. These signals are used to align the cavity mirrors at the end stations $\mathrm{PC}_{1}$ and $\mathrm{RC}_{2}$.

the number of substrates which shift the beam to the right, assuming that the substrates are equal in thickness and in material, and that the angle of incidence is the same. For example, the HET design uses two substrates that shift the beam left and two that shift it right; all at $35^{\circ}$ angle of incidence and all made from fused silica. According to the vendor, the substrates are $9.5 \mathrm{~mm}$ thick with a tolerance of $[+0,-0.5 \mathrm{~mm}]$. This results in a worst case lateral shift of $\sim 200 \mu \mathrm{m}$.

Based on these numbers, the uncertainty in the resulting mode mismatch between the PC transmitted field and the axion field for both degrees of freedom will be below $0.4 \%$ which will allow us to use the PC transmitted field to verify the overall alignment of the axion mode into the RC at the $95 \%$ level.

\subsubsection{Central Mirror Alignment}

The central optical bench (COB) is a critical piece of the optical system. Its main role is to ensure that the two cavities maintain their relative alignment during the science runs. This requires that the surfaces of the flat cavity mirrors are parallel to each other, and that the positions of the eigenmodes of the two cavities are in line with each other. The COB uses no active alignment system; instead, we rely on its passive stability, and control loops that stabilize the spot positions on the COB mirrors. Open shutter measurements will be used to quantify the overall misalignment.

The COB is constructed from a single aluminum plate on which all mirrors, beam splitters, and waveplates, as well as position sensors are either mounted directly or through additional ULE base plates [1] using, in both cases, ultra-stable optical mounts. Tests with an autocollimator have shown that a prototype $\mathrm{COB}$ was capable of maintaining a long term alignment stability of $2 \mu \mathrm{rad}$ over one week in air with measured thermal alignment coefficients of $\approx 4 \mu \mathrm{rad} / \mathrm{K}$ in pitch and $\approx 1 \mu \mathrm{rad} / \mathrm{K}$ in yaw $[35$. The air conditioning system of the cleanroom has been designed to maintain an $0.1 \mathrm{~K}$ absolute temperature stability which would, in principle, eliminate any relevant misalignment. However, the impact of the heating of $\mathrm{PC}_{2}$ by the cavity internal field on the alignment still needs to be evaluated during the commissioning of the experiment.

The alignment process of the $\mathrm{COB}$ starts with the two cavity mirrors $\mathrm{PC}_{2}$ and $\mathrm{RC}_{1}$. We will use two counter propagating laser beams, likely derived from a single HeNe laser, and two position sensors with $\mu \mathrm{m}$ resolution about $1 \mathrm{~m}$ away from the COB. The sensors will initially mark the forward propagating positions of the two beams and then act as references for when the cavity mirrors are installed and the reflected beams become available. The transmissivity of the cavity mirrors will allow us to observe the beams in transmission and reflection by simply blocking one of them. This method should be sufficient to achieve an initial alignment of the surface normals of $\mathrm{PC}_{2}$ and $\mathrm{RC}_{1}$ of better than $\delta \theta<3 \mu \mathrm{rad}$ taking also into account the $1 \mu \mathrm{rad}$ residual angular refraction caused by the wedge angles of the cavity mirrors. 


\subsubsection{End Mirror Alignment}

As Figure 3 shows, the COB will also host two in-vacuum QPDs that will monitor the position of the cavity eigenmodes with respect to the COB by making DC differential measurements of the light incident on their quandrants. These QPDs are optimized to sense the position of the $6 \mathrm{~mm}$ radius beam with sub $100 \mathrm{\mu m}$ precision $[36$. Assuming that the components on the COB remain stationary, the positions of the eigenmodes on the flat cavity mirrors $\mathrm{PC}_{2}$ and $\mathrm{RC}_{1}$, and in extension on the two QPDs, only depend on the orientations of the curved cavity mirrors $\mathrm{PC}_{1}$ and $\mathrm{RC}_{2}$, respectively. The differential signals from the QPDs will be fed back to active alignment stages that are capable of controlling the pitch and yaw of the curved cavity end mirrors.

The transversal shift $\delta x$ between the two eigenmodes is therefore expected to be below $200 \mu \mathrm{m}$. The resulting mode matching losses due to static cavity misalignments should be

$$
1-\eta_{\mathrm{TM}} \approx\left(\frac{\delta x}{w_{0}}\right)^{2}+\left(\frac{\delta \theta}{\theta_{\text {Div }}}\right)^{2}<0.5 \%
$$

on the same order as the matching between the axion field and the PC transmitted field after traversing the $\mathrm{COB}$ optics, leaving significant margin for systematic errors and drifts.

\section{Summary and Conclusion}

This paper describes the core components and the design of the ALPS II experiment and represents the most detailed plan up to now, to maintain the coherence and spatial overlap of two high finesse optical cavities for a LSW experiment. Upon reaching design sensitivity ALPS II will become the most sensitive LSW experiment to date by three orders of magnitude, and the innovations in the optical system alone account for nearly two orders of magnitude in sensitivity gains. Additional gains in sensitivity will come from the long magnetic field length and improvements in detector technologies [22, 25, 1].

One of the main challenges for the optical system will be to maintain and verify the coherence of the axion field with respect to length of the $\mathrm{RC}$, as well as the alignment and mode matching between the spatial modes of the axion field and the RC eigenmode. As described in this paper, we will take great care that the spatial mode of the transmitted light from the $\mathrm{PC}$ that is incident on the $\mathrm{RC}$ is an accurate representation of the axion mode. This will allow us to quantify the coherence and mode matching of the axion field with respect to the RC. The length and alignment sensing system for the lasers and the cavities is based on PDH and DWS, well established phase sensing schemes with sufficient sensitivity to monitor all relevant degrees of freedom. Additionally, we developed and tested different actuators which should have enough range and bandwidth to operate ALPS II in the HERA tunnels.

We also discussed our plans to employ two different schemes to detect the regenerated photon signal. The first one is the HET and is described in detail in an accompanying paper. The second scheme uses a TES, and its optical design is currently being finalized and will be implemented following the HET science runs. The experiment itself is presently under construction and aiming for a first science run in 2021. Once fully operational, the optical system should allow ALPS II to be able to detect axions with a coupling constant as low as $g_{a \gamma \gamma}=2 \times 10^{-11} / \mathrm{GeV}$ using 20 days of valid science data.

\section{Acknowledgments}

The work is supported by the Deutsche Forschungsgemeinschaft through SFB 676 and project grant WI 1643/2-1, by the German Volkswagen Stiftung, the National Science Foundation under grant 
1802006, the Heising Simons Foundation under award 2015-154, and the Science and Technologies Facilities Council.

\section{References}

[1] Ayman Hallal, Giuseppe Messineo, Mauricio Diaz Ortiz, Joseph Gleason, Harold Hollis, David B Tanner, Guido Mueller, and Aaron D Spector. Design of the heterodyne detection system for ALPS II. arXiv 2010:3386532, 2020.

[2] Robin Bähre, Babette Döbrich, Jan Dreyling-Eschweiler, Samvel Ghazaryan, Reza Hodajerdi, Dieter Horns, Friederike Januschek, E-A Knabbe, Axel Lindner, Dieter Notz, et al. Any light particle search IItechnical design report. J. Instrum., 8(09):T09001, 2013.

[3] Klaus Ehret, Maik Frede, Samvel Ghazaryan, Matthias Hildebrandt, Ernst-Axel Knabbe, Dietmar Kracht, Axel Lindner, Jenny List, Tobias Meier, Niels Meyer, et al. New ALPS results on hidden-sector lightweights. Phys. Lett., 689(4-5):149-155, 2010.

[4] R Ballou, G Deferne, M Finger Jr, M Finger, L Flekova, J Hosek, S Kunc, K Macuchova, KA Meissner, P Pugnat, et al. New exclusion limits on scalar and pseudoscalar axionlike particles from light shining through a wall. Phys. Rev. D, 92(9):092002, 2015.

[5] Roberto D Peccei and Helen R Quinn. Cp conservation in the presence of pseudoparticles. Phys. Rev. Lett., 38(25):1440, 1977.

[6] Steven Weinberg. A new light boson? Phys. Rev. Lett., 40(4):223, 1978.

[7] Frank Wilczek. Problem of strong p and t invariance in the presence of instantons. Phys. Rev. Lett., 40(5):279, 1978.

[8] Manuel Meyer, Dieter Horns, and Martin Raue. First lower limits on the photon-axion-like particle coupling from very high energy gamma-ray observations. Phys. Rev. D, 87(3):035027, 2013.

[9] Maurizio Giannotti, Igor Irastorza, Javier Redondo, and Andreas Ringwald. Cool WISPs for stellar cooling excesses. J. Cosmol. Astropart. P., 2016(05):057, 2016.

[10] Holger Gies, Joerg Jaeckel, and Andreas Ringwald. Polarized light propagating in a magnetic field as a probe for millicharged fermions. Phys. Rev. Lett., 97(14):140402, 2006.

[11] Masaharu Tanabashi, K Hagiwara, K Hikasa, Katsumasa Nakamura, Y Sumino, F Takahashi, J Tanaka, K Agashe, G Aielli, Claude Amsler, et al. Review of particle physics. Phys. Rev. D, 98(3):030001, 2018.

[12] V Anastassopoulos, S Aune, K Barth, A Belov, H Bräuninger, Giovanni Cantatore, JM Carmona, JF Castel, SA Cetin, F Christensen, et al. New CAST limit on the axion-photon interaction. Nat. Phys., 13(6):584, 2017.

[13] E Armengaud, FT Avignone, M Betz, P Brax, P Brun, Giovanni Cantatore, JM Carmona, GP Carosi, F Caspers, S Caspi, et al. Conceptual design of the international axion observatory (IAXO). J. Instrum., 9(05):T05002, 2014.

[14] N Du, N Force, R Khatiwada, E Lentz, R Ottens, LJ Rosenberg, Gray Rybka, G Carosi, $\mathrm{N}$ Woollett, D Bowring, et al. Search for invisible axion dark matter with the axion dark matter experiment. Phys. Rev. Lett., 120(15):151301, 2018. 
[15] P Brun, A Caldwell, L Chevalier, G Dvali, P Freire, E Garutti, S Heyminck, J Jochum, S Knirck, M Kramer, et al. A new experimental approach to probe QCD axion dark matter in the mass range above $40 \mu \mathrm{eV}$. Eur. Phys. J. C, 79(3):1-16, 2019.

[16] K Van Bibber, NR Dagdeviren, SE Koonin, AK Kerman, and HN Nelson. Proposed experiment to produce and detect light pseudoscalars. Phys. Rev. Lett., 59(7):759, 1987.

[17] P Sikivie, DB Tanner, and Karl van Bibber. Resonantly enhanced axion-photon regeneration. Phys. Rev. Lett., 98(17):172002, 2007.

[18] Javier Redondo and Andreas Ringwald. Light shining through walls. Contemp. Phys., 52(3):211-236, 2011.

[19] F Hoogeveen and Thomas Ziegenhagen. Production and detection of light bosons using optical resonators. Nucl. Phys., 358(1):3-26, 1991.

[20] Yukio Fukuda, Toshiro Kohmoto, Shin-ichi Nakajima, and Masakuzu Kunitomo. Production and detection of axions by using optical resonators. Prog. Cryst. Growth Ch., 33(1-3):363-366, 1996.

[21] Jan Hendrik Põld and Hartmut Grote. ALPS II design requirements document. 2019.

[22] Clemens Albrecht, Serena Barbanotti, Heiko Hintz, Kai Jensch, Ronald Klos, Wolfgang Maschmann, Olaf Sawlanski, Matthias Stolper, and Dieter Trines. Straightening of superconducting HERA dipoles for the any-light-particle-search experiment ALPS II. arXiv 2004.13441, 2020 .

[23] Paola Arias, Joerg Jaeckel, Javier Redondo, and Andreas Ringwald. Optimizing light-shiningthrough-a-wall experiments for axion and other weakly interacting slim particle searches. Phys. Rev. D, 82(11):115018, 2010.

[24] Zachary R Bush, Simon Barke, Harold Hollis, Aaron D Spector, Ayman Hallal, Giuseppe Messineo, DB Tanner, and Guido Mueller. Coherent detection of ultraweak electromagnetic fields. Phys. Rev. D, 99(2):022001, 2019.

[25] Jan Dreyling-Eschweiler, Noemie Bastidon, Babette Döbrich, Dieter Horns, Friederike Januschek, and Axel Lindner. Characterization, $1064 \mathrm{~nm}$ photon signals and background events of a tungsten TES detector for the ALPS experiment. J. Mod. Optic., 62(14):1132-1140, 2015.

[26] Fabian Thies, Nina Bode, Patrick Oppermann, Maik Frede, Bastian Schulz, and Benno Willke. Nd: YVO 4 high-power master oscillator power amplifier laser system for second-generation gravitational wave detectors. Opt. Lett., 44(3):719-722, 2019.

[27] RWP Drever, John L Hall, FV Kowalski, J_ Hough, GM Ford, AJ Munley, and H Ward. Laser phase and frequency stabilization using an optical resonator. Appl. Phys. B, 31(2):97-105, 1983.

[28] Eric D Black. An introduction to Pound-Drever-Hall laser frequency stabilization. Am. J. Phys., 69(1):79-87, 2001.

[29] Euan Morrison, Brian J Meers, David I Robertson, and Henry Ward. Automatic alignment of optical interferometers. Appl. Opt., 33(22):5041-5049, 1994.

[30] A Buikema, C Cahillane, GL Mansell, CD Blair, R Abbott, C Adams, RX Adhikari, A Ananyeva, S Appert, K Arai, et al. Sensitivity and performance of the advanced ligo detectors in the third observing run. arXiv 2008.01301, 2020. 
[31] Lamar Glover, Michael Goff, Jignesh Patel, Innocenzo Pinto, Maria Principe, Travis Sadecki, Richard Savage, Ethan Villarama, Eddy Arriaga, Erik Barragan, et al. Optical scattering measurements and implications on thermal noise in gravitational wave detectors test-mass coatings. Phys. Lett., 382(33):2259-2264, 2018.

[32] Walter Winkler, Karsten Danzmann, Albrecht Rüdiger, and Roland Schilling. Heating by optical absorption and the performance of interferometric gravitational-wave detectors. Phys. Rev. A, 44(11):7022, 1991.

[33] James I Thorpe, K Numata, and J Livas. Laser frequency stabilization and control through offset sideband locking to optical cavities. Opt. Express, 16(20):15980-15990, 2008.

[34] Jan H Põld and Aaron D Spector. Demonstration of a length control system for ALPS II with a high finesse $9.2 \mathrm{~m}$ cavity. Eur. Phys. J. TI, 7(1):1-9, 2020.

[35] Soham Kulkarni, Ada Umińska, Joseph Gleason, Simon Barke, Reid Ferguson, Jose Sanjuán, Paul Fulda, and Guido Mueller. Ultrastable optical components using adjustable commercial mirror mounts anchored in a ULE spacer. Appl. Opt., 59(23):6999-7003, 2020.

[36] Li-Wei Wei, Kanioar Karan, and Benno Willke. Optics mounting and alignment for the central optical bench of the dual cavity enhanced light-shining-through-a-wall experiment alps ii. Appl. Opt., 59(28):8839-8847, Oct 2020. 